\title{
Next-Generation Gravity Missions: Sino-European Numerical Simulation Comparison Exercise
}

\author{
Roland Pail ${ }^{1, *}$, Hsien-Chi Yeh ${ }^{2}$, Wei Feng ${ }^{3}{ }^{-}$, Markus Hauk ${ }^{1}$, Anna Purkhauser ${ }^{1}$, \\ Changqing Wang ${ }^{3}$, Min Zhong ${ }^{3}$, Yunzhong Shen ${ }^{4}$, Qiujie Chen ${ }^{4}$, Zhicai Luo ${ }^{5}$, Hao Zhou ${ }^{5}(0)$ \\ Bingshi Liu ${ }^{6}$, Yongqi Zhao ${ }^{6}$, Xiancai Zou ${ }^{6}$, Xinyu Xu ${ }^{6}{ }^{\infty}$, Bo Zhong ${ }^{6}$, Roger Haagmans ${ }^{7}$ \\ and Houze $\mathrm{Xu}^{3}$ \\ 1 Institute of Astronomical and Physical Geodesy, Technical University of Munich, 80333 Munich, Germany; \\ markus.hauk@tum.de (M.H.); anna.purkhauser@tum.de (A.P.) \\ 2 School of Physics and Astronomy, Sun Yat-sen University (Zhuhai Campus), Zhuhai 519082, China; \\ yexianji@mail.sysu.edu.cn \\ 3 State Key Laboratory of Geodesy and Earth's Dynamics, Institute of Geodesy and Geophysics, APM, \\ Chinese Academy of Sciences, Wuhan 430077, China; fengwei@whigg.ac.cn (W.F.); \\ whiggsdkd@whigg.ac.cn (C.W.); zmzm@whigg.ac.cn (M.Z.); hsuh@whigg.ac.cn (H.X.) \\ 4 College of Surveying and Geo-informatics, Tongji University, Shanghai 200092, China; \\ yzshen@tongji.edu.cn (Y.S.); qiujie.chen@uni-bonn.de (Q.C.) \\ 5 Institute of Geophysics and PGMF, Huazhong University of Science and Technology, Wuhan 430074, China; \\ zcluo@hust.edu.cn (Z.L.); zhouh@hust.edu.cn (H.Z.) \\ 6 School of Geodesy and Geomatics, Wuhan University, Wuhan 430079, China, and Key \\ Laboratory of Geospace Environment and Geodesy, Ministry of Education, Wuhan 430079, China; \\ bs.liu@whu.edu.cn (B.L.); yqzhao@whu.edu.cn (Y.Z.); xczou@sgg.whu.edu.cn (X.Z.); \\ xyxu@sgg.whu.edu.cn (X.X.); bzhong@sgg.whu.edu.cn (B.Z.) \\ 7 ESA-European Space Agency, 2201 AZ Noordwijk, The Netherlands; roger.haagmans@esa.int \\ * Correspondence: roland.pail@tum.de; Tel.: +49-89-289-23199
}

Received: 10 October 2019; Accepted: 11 November 2019; Published: 13 November 2019

\begin{abstract}
Temporal gravity retrieval simulation results of a future Bender-type double pair mission concept, performed by five processing centers of a Sino-European study team, have been inter-compared and assessed. They were computed in a synthetic closed-loop simulation world by five independent software systems applying different gravity retrieval methods, but were based on jointly defined mission scenarios. The inter-comparison showed that the results achieved a quite similar performance. Exemplarily, the root mean square (RMS) deviations of global equivalent water height fields from their true reference, resolved up to degree and order 30 of a 9-day solution, vary in the order of $10 \%$ of the target signal. Also, co-estimated independent daily gravity fields up to degree and order 15, which have been co-estimated by all processing centers, do not show large differences among each other. This positive result is an important pre-requisite and basis for future joint activities towards the realization of next-generation gravity missions.
\end{abstract}

Keywords: next-generation gravity mission; temporal gravity field; numerical closed-loop simulation; satellite mission constellations; mass transport

\section{Introduction}

Next Generation Gravity Missions (NGGMs), expected to be launched in the midterm future, have set high anticipations for an enhanced monitoring of mass transport in the Earth system, establishing that their products are applicable to new scientific fields and serving societal needs. Past and current gravity missions such as Gravity Recovery and Climate Experiment (GRACE, [1]) and 
GRACE Follow-On [2] have improved our understanding of many mass change processes, such as the global water cycle, ice mass melting of ice sheets and glaciers, changes in ocean mass closely related to eustatic sea level rise, which are subtle indicators of climate change, but also gravity changes related to solid Earth processes such as big earthquakes or glacial isostatic adjustment (GIA). The Gravity field and steady-state Ocean Circulation Explorer (GOCE) mission [3] has improved our knowledge of long-term mass distribution and has provided the physical reference surface of the geoid, with a resolution down to $70-80 \mathrm{~km}$.

The main objective of NGGMs is not only the continuation and extension of existing mass transport time series, but also a significant gain in spatial and temporal resolution. Correspondingly, the science and user needs of NGGMs were defined in [4]. In several previous studies, different mission concepts have been studied in detail, with emphasis on orbit design and resulting spatial-temporal ground track pattern, enhanced processing and parameterization strategies, and improved post-processing/filtering strategies in order to reduce temporal aliasing effects, which are one of the main error sources of current temporal gravity field solutions [5]. The typical GRACE-type concept of two satellites (satellite pair) following each other on the same orbit with an inter-satellite distance of about $200 \mathrm{~km}$ observes only the along-track component of the Earth's gravity field, and therefore leads to a very anisotropic error structure and the typical striping patterns in the resulting temporal gravity solutions. An alternative mission concept is a satellite pair in pendulum configuration, where the trailing satellite performs a pendulum motion with respect to the leading satellite, thus observing not only the along-track, but also parts of the cross-track component. This can be realized by a shift of the right-ascension of the satellites of the second pair, resulting in a shifted orbit plane of the second satellite pair compared to the first one. Based on this concept, in 2010 the mission proposal "e.motion —Earth System Mass Transport Mission" [6] was submitted in response to ESA's Earth Explorer 8 call. Another promising mission scenario is a double-pair constellation being composed of two in-line pairs, the so-called Bender configuration [7]. It is composed of a polar pair similar to the GRACE-type concept, and an inclined pair with an inclination of 65-70 degrees. An example of the resulting ground-track pattern of such a Bender constellation is shown in Figure 1.

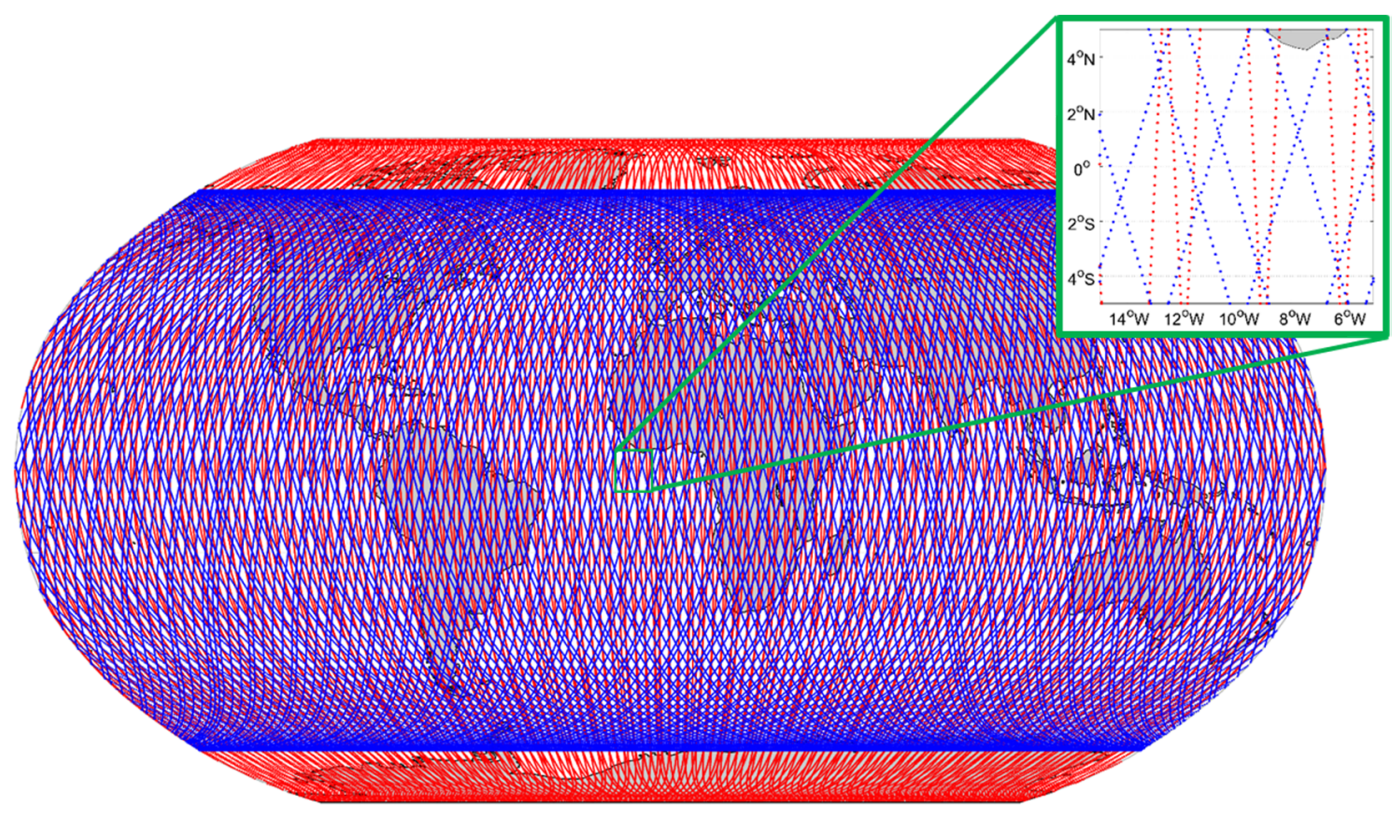

Figure 1. Ground track pattern of Bender configuration of Scenario A (cf. Table 1). The red curve shows the 9-day near-repeat ground track pattern of the polar satellite pair, and the blue curve the ground track of the inclined pair with an inclination of $70^{\circ}$. The zoom-in illustrates the resulting regular spatial sampling pattern as well as the fact that the direction of the inter-satellite ranges is different. 
Table 1. Main orbit parameters of numerical simulation scenarios.

\begin{tabular}{cccccc}
\hline $\begin{array}{c}\text { Simulation } \\
\text { Scenario }\end{array}$ & $\begin{array}{c}\text { Semi-major } \\
\text { Axis Minus } \\
\mathbf{6 3 7 8} \mathbf{~ k m}\end{array}$ & Inclination $\left(^{\circ}\right)$ & $\begin{array}{c}\text { Cycle/Retrieval } \\
\text { Period (days) }\end{array}$ & Start Epoch & $\begin{array}{c}\text { Drift Rate } \\
(\% / \text { cycle) }\end{array}$ \\
\hline A & $\sim 355 / \sim 390$ & $70 / 89$ & 9 & 1 Jan 2002 & 1.5 \\
B & $\sim 355 / \sim 340$ & $70 / 89$ & 7 & 1 Jan 2002 & 1.3 \\
\hline
\end{tabular}

Extensive numerical simulations have shown that this set-up also results in a significantly improved isotropy and reduction of stripes, e.g., [8,9], which is mainly due to the different directions of inter-satellite ranging observations, and the different orbital frequencies of the two pairs. Based on this concept, in 2016 e.motion ${ }^{2}$ was proposed as ESA Earth Explorer 9 mission [10]. Another innovative observation concept is high-precision high-low inter-satellite ranging, which was proposed as the MOBILE mission in response to ESA's Earth Explorer 10 call, e.g., [11] and [12]. On US side, the United States National Academies of Sciences, Engineering, and Medicine published the decadal strategy for Earth observation from space [13], where mass change was identified as one of the top five designated observables to be implemented by future US Earth observation missions, in order to ensure continuity and enable long-term mass budget analyses of the Earth system.

A NGGM concept was also proposed in China, and the key technologies have been developed to meet the requirements of NGGM [14-16]. The objectives of Chinese NGGM are not only to perform global gravity measurements, but also to verify the maturity of key technologies required for space-based gravitational-wave detection [17] to a certain level of precision. The scientific objectives and the system requirements of Chinese NGGM are under investigation in these years. This mission is expected to be approved soon, and is planned to launch in the middle 2020s.

The existence of this study roots back to a Round Table China-Europe meeting on Satellite Gravity Exploration, which took place in September 2013 in Beijing. There it was agreed to evaluate options for further joint activities on NGGMs, especially science studies on numerical mission simulations. This was eventually realized in the frame of the European Space Agency's "Additional Constellation and Scientific Analysis Studies of the Next Generation Gravity Mission (ADDCON)" study. ESA's ADDCON study team and a Chinese study team joined forces in an initiative to perform numerical studies on NGGMs based on double-pair and multi-pair constellations of GRACE/GRACE-FO-type satellites.

A main prerequisite for the performance assessment and interpretation of joint numerical simulations is to ensure comparability of the results of the contributing groups. Therefore, it was decided early in the project to jointly set up common test scenarios and corresponding data sets, which shall be processed by all contributing processing centers. This paper reports on results of this inter-comparison exercise. The contributing processing centers are Technical University of Munich (TUM), Institute of Geodesy and Geophysics, Chinese Academy of Sciences (IGG-CAS), Tongji University (Tongji), Wuhan University (WHU), and Huazhong University of Science and Technology (HUST).

The paper is structured as follows. Chapter 2 describes the basic idea of a numerical closed-loop NGGM simulation, and describes the main input data, which have been jointly used by all groups. Chapter 3 provides an overview of different methods applied by the study teams. Chapter 4 reports the numerical simulation results, their comparison, assessment, and inter-comparison. The final Chapter 5 contains main conclusions as well as an outlook to future work.

\section{Closed-Loop Simulations and Input Data}

The basis of the inter-comparison exercise are so-called numerical closed-loop simulations. Compared to real-data processing, the advantage of these closed-loop simulations, which are performed in a completely synthetic simulation world, is that the truth is perfectly known. Therefore, the effect of any data set, instrument noise assumption, or processing strategy is directly reflected by deviation of the final result from the "true world", and can be studied separately from all other error contributors to the total error budget. 
Figure 2 shows an overview of the numerical simulation set-up. The simulation starts with the definition of the "true world" (upper left), using force models for the static gravity field as well as tidal and non-tidal temporal variations. All gravity-related models are parameterized as coefficients of a spherical harmonic $(\mathrm{SH})$ series expansion. In parallel, specific orbit constellations and the corresponding orbit parameters have to be defined, in our case Bender-type double pairs. The corresponding orbits are generated by numerical integration. Based on the a-priori force models, error-free observations are computed along the orbit tracks, with a sampling period of $5 \mathrm{~s}$. They are then superimposed by instrument errors with pre-specified spectral noise characteristics, resulting in "true observations", which mimic as realistically as possible the real observations. On the other hand, a reference world is built up (upper right). This is necessary because the functional model of gravity field estimation from low-low inter-satellite ranging observations is highly non-linear, and this requires a Taylor point as approximate prior knowledge. In the same way as for the "true observations", so-called "reference observations" are computed, which are based on force models that are usually different from those used for the "true world" scenario. The main goal of the gravity field determination is then to estimate residuals with respect to these a-priori models. Adding the residuals to the a-priori models should result in the ideal case (no errors in the system) in the true world models. In the case that errors do exist, such as instrument errors, but also aliasing errors due to the specific space-time sampling properties of the chosen orbit constellation, the estimated gravity field will deviate from the true world, and thus provides a clear hint on the achievable performance of the specific mission design.

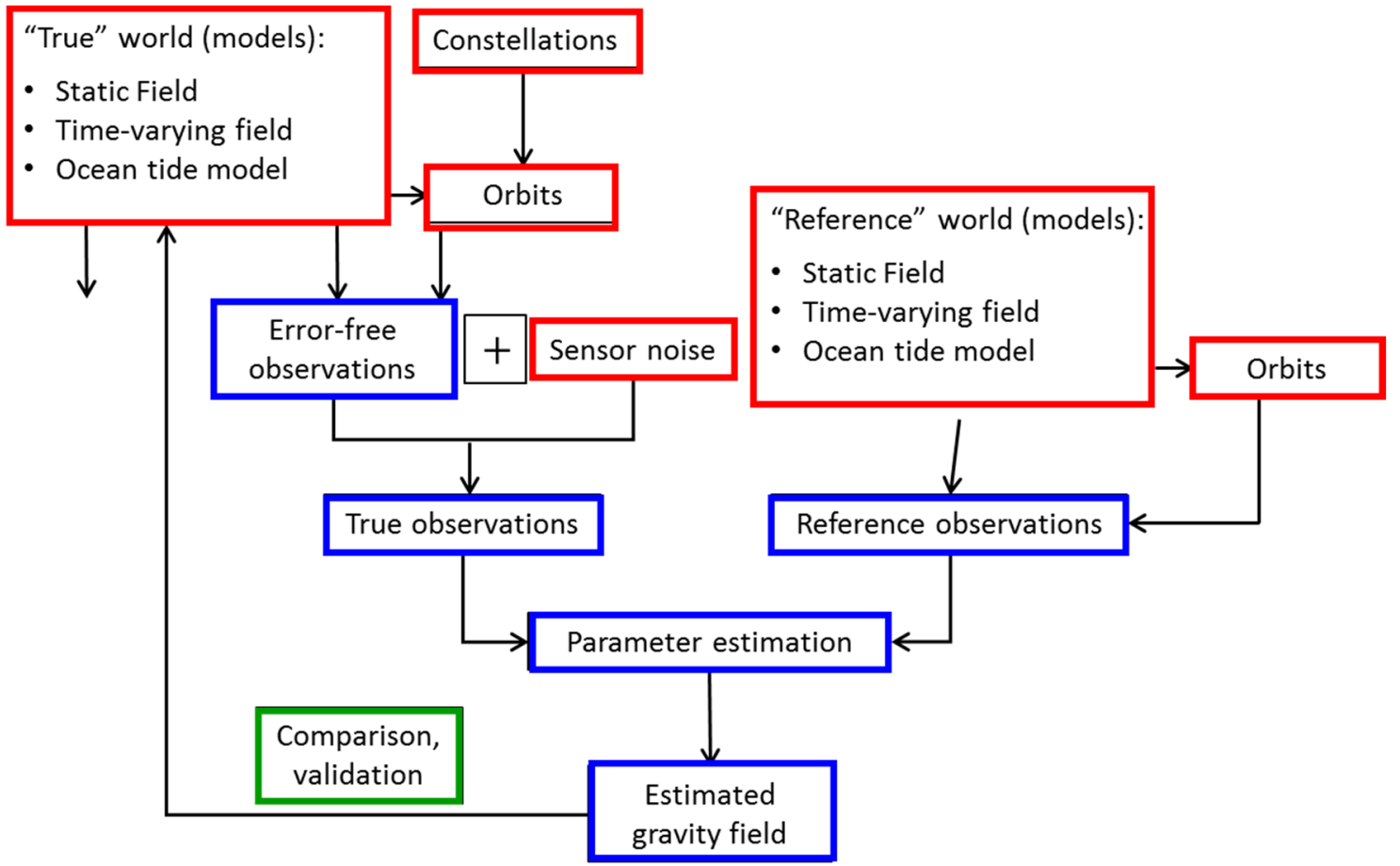

Figure 2. Scheme of a closed-loop simulation set-up. The red blocks are related to orbit design and input data generation, the blue blocks are tightly linked to the gravity parameter estimation process, while the green block refers to the comparison and validation of the resulting gravity models.

For the inter-comparison exercise, two Bender double-pair scenarios have been defined (Table 1). They are based on extensive prior studies on optimum orbit design of double-pair configuration with the goal to optimize the space-time sampling. The two scenarios A and B mainly differ in the orbit altitude of the polar satellite (390 vs. $340 \mathrm{~km}$ ) and their repeat cycle ( 9 vs. 7 days). The orbits were designed such that ground tracks of the polar and inclined satellite pair drift at the same rate within the sub-cycle period, and (b) the retrieval period is always equal to the length of the sub-cycle, so that the gravity field retrieval is always supported by the densest possible ground track pattern. As a side 
aspect, these drifting orbits have the additional advantage that they produce a dense ground-track distribution over longer periods of time, which might improve the spatial resolution for the gravity retrieval of long-term static gravity solutions.

Table 2 provides an overview of the force and noise models of the true and reference world. Static, tidal, and non-tidal gravity models are used up to SH degree and order (d/o) 120.

Table 2. Force and noise models of the "true" and "reference" world used in the Next Generation Gravity Mission (NGGM) simulations.

\begin{tabular}{ccc}
\hline Model & True World & Reference World \\
\hline Static gravity field & GOCO05s [18] & GOCO05s \\
Non-tidal time-variable gravity field & ESA AOHIS [19] & - \\
Ocean tides & EOT11a [20] & GOT4.7 [21] \\
Inter-Satellite Link (ISL) noise & Equation (1) & - \\
Accelerometer (ACC) noise & Equations (2) and (3) & - \\
Star tracker (STR) noise & - & - \\
Orbit noise & 1 cm white noise & - \\
\hline
\end{tabular}

The ISL is assumed to be realized by a Laser Ranging Interferometer (LRI). The assumed spectral noise behavior of the LRI in terms of range rate is expressed by the following Amplitude Spectral Density (ASD) [22]:

$$
d_{\text {range-rates }}=2 \times 10^{-8} \times 2 \pi f \times \sqrt{\left(\frac{10^{-2} \mathrm{~Hz}}{f}\right)^{2}+1} \frac{\mathrm{m}}{\mathrm{s} \sqrt{\mathrm{Hz}}}
$$

where $f$ is the linear frequency. The resulting error spectrum is shown in Figure 3a (dark blue curve), and the corresponding noise realization in light blue color.

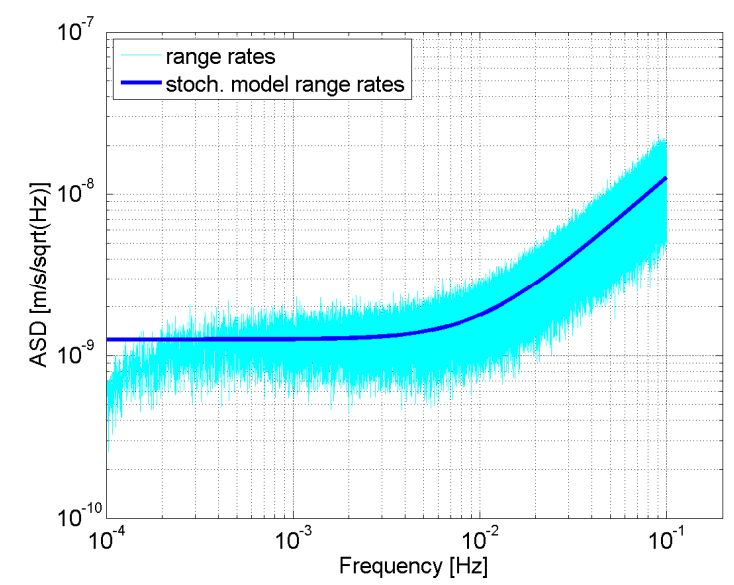

(a)

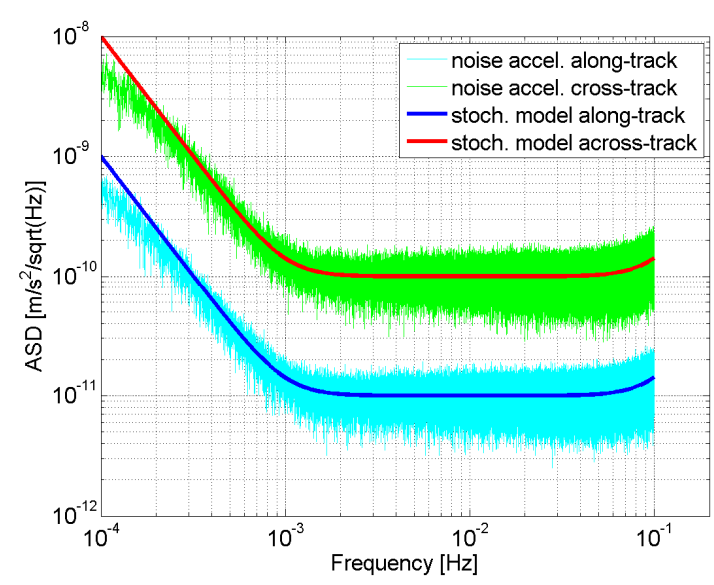

(b)

Figure 3. Amplitude spectral density (ASD) of (a) relative distant measurement errors in terms of range-rates, and (b) accelerometer errors. The dark blue and red curves show the analytical noise models in accordance with Equations (1) to (3), and the light blue and green curves the corresponding noise realizations, which have been generated scaling the spectrum of normally distributed random time-series with their individual spectral model.

The non-gravitational forces are typically sensed by the on-board accelerometers located in the center-of-mass of the satellite. In our simulations, an electrostatic accelerometer is assumed with two high-sensitive axes oriented in the flight direction (largest signal) and radial direction, and one 
low-sensitive axis in the cross-track direction (see Figure 3b). The accuracy level in terms of accelerations is expressed by [22]:

$$
\begin{gathered}
d_{\text {acc. along }}=d_{\text {acc. radial }}=10^{-11} \sqrt{\left(\frac{10^{-3} \mathrm{~Hz}}{f}\right)^{4} /\left(\left(\frac{10^{-5} \mathrm{~Hz}}{f}\right)^{4}+1\right)+1+\left(\frac{f}{10^{-1} \mathrm{~Hz}}\right)^{4}} \frac{\mathrm{m}}{\mathrm{s}^{2} \sqrt{\mathrm{Hz}}} \\
d_{\text {acc. across }}=10 \times d_{\text {acc. along, }}
\end{gathered}
$$

It has a noise level of $10^{-11} \mathrm{~m} / \mathrm{s}^{2}$ in the measurement bandwidth, and a $1 / f^{2}$ slope at frequencies from $10^{-3} \mathrm{~Hz}$ and lower. Equation (3) expresses the fact that electrostatic accelerometers, as they have been used in all previous and current satellite gravity missions, usually have two high-sensitive axes and one less-sensitive axis. In our set-up, we assume a degraded performance of the less-sensitive axis by one order of magnitude, and that it is oriented in an across-track direction. In this study, we further assume that the attitude, which is usually a minor error contributor compared to ISL and ACC, is known perfectly. Orbit errors are simulated by white noise with a standard deviation of $1 \mathrm{~cm}$. The generation of the ISL and ACC noise time-series was done by scaling the spectrum of normally distributed random time-series with their individual spectral analytical noise model, as given in Equations (1) to (3).

\section{Methods}

The five contributing processing centers used their specific software systems for NGGM simulation and gravity field recovery. However, as a joint spatial-temporal parametrization, low-degree daily gravity field parameters were co-estimated together with the higher-degree temporal gravity solution, where the latter covers a retrieval period of 9 days (Scenario A) and 7 days (Scenario B). This co-estimation was first proposed by [23] and investigated in detail in [9]. There it could be shown that by this "Wiese parameterization" temporal aliasing can be reduced significantly, because short-term temporal gravity signals (mainly atmosphere and ocean signals), which would alias in standard processing into the solutions, are explicitly estimated, thus avoiding their aliasing effect. On the one hand this "self-dealiasing" approach improves the gravity field solution over the whole spectral range, and on the other hand, as a positive side effect daily gravity field products, which are independent of each other, are produced. This might be an important aspect for operational service applications, where usually a high temporal resolution and short latencies are required [24].

In all five processing centers, the non-tidal gravity field signals along the orbits were derived from the ESA Earth System Model (ESM, [19]) for all five components: atmosphere (A), ocean (O), hydrology (H), ice (I), and solid Earth (S), together abbreviated by AOHIS. Based on the 6-hourly AOHIS input products, the signals at certain measurement epochs were derived by linear interpolation. In the recovery, the full AOHIS signal was estimated, i.e., no a-priori non-tidal de-aliasing is performed, which is possible due to the co-parameterization of daily gravity field parameters [8,9]. Therefore, also no additional errors were assumed for the AOHIS signal. The true world is composed of this AOHIS signal itself, and the reference world does not contain any non-tidal temporal gravity signal (cf. Table 2).

In the following, we describe briefly the different methods applied by the five processing centers. Detailed information can be found in the cited references.

TUM: All simulations were executed with a full-scale numerical mission simulator $[25,26]$, which has already been successfully applied in real data applications to recover satellite-only gravitational field models for CHAMP (Challenging Mini-Satellite Payload), GRACE and GOCE. The simulation environment is based on numerical orbit integration following a multistep method [27], which applies a modified divided difference form of the Adams-Bashforth-Moulton Predict-Evaluate-Correct-Evaluate (PECE) formulas and local extrapolation. The adopted gravity field approach is based on a modification of the integral equation approach from [28] where the orbit is divided into continuous short arcs of $6 \mathrm{~h}$ length. The position vectors at the arc node points are set up as unknown parameters, which are estimated together with the gravity field coefficients. As a stochastic 
observation model, autocovariances computed from an auto-regressive moving average (ARMA) filter model, approximating the spectral characteristics of the residuals of instrument errors, are used [9].

IGG-CAS: Simulation work was done with a full numerical mission simulator, which is modified from the software tool developed in IGG-CAS for recovering satellite-only gravitational field models from GRACE Level1b observations [29]. The simulation environment is based on numerical orbit integration applying the multistep Gauss-Jackson method [30], which uses start-up value using Runge-Kutta single step integral method. The gravity field recovery is based on the variational equation approach or dynamic method, where the orbit is divided into continuous arcs of $6 \mathrm{~h}$ length, and initial state vectors at the beginning of the arc are set up as unknown parameters, which are estimated together with the gravity field coefficients. The 1-cpr effect in the residuals of range-rate observation are modelled [31], which are also estimated together with the gravity field coefficients.

Tongji: The simulations were carried out by using the Satellite Gravimetry Analysis Software (SAGAS) developed by Tongji University [32-35], which has been successfully applied to determine gravity field models from GRACE Level-1b observations. The simulation environment is based on numerical orbit integration, where Adams and Kiogh-Shampine-Gordon (KSG) numerical integration methods are jointly used. The adopted gravity field approach is the modified short-arc approach [32-34], where the observation equation is directly linearized with respect to the real orbit observations and the integral arc is divided into continuous short arcs of $2 \mathrm{~h}$ in length. Non-conservation acceleration observation errors are estimated together with the gravity field coefficients according to [33,34]. Variance-covariance matrices for both orbit and range-rate observations are constructed from residuals of the instrument errors on the basis of [35].

WHU: WHU team has developed a software platform which was used in the real data processing of the modern satellite gravity missions such as GRACE and GOCE [36,37]. For the current work on the evaluation of the NGGM schemes described in this paper, the brute-force dynamic method was selected as the basic approach in the numerical simulations [38]. For the non-conservative forces there was only accelerometer noise provided, so the orbit was integrated with the non-gravitational forces being constant zeros. In the parameter estimation step, the arc length is adjusted to $1.5 \mathrm{~h}$ for an optimal estimation. The initial satellite states of every arc and low degree geo-potential coefficients up to $\mathrm{d} / \mathrm{o} 15$ were processed as the local parameters, and the other geopotential coefficients to degree 70 were estimated as the global ones [23]. Regarding the stochastic model, a filtering strategy of processing band-limited measurements proposed by Schuh [39] was applied to deal with the colored observation noise.

HUST: All simulation works were implemented with a full numerical mission simulator, which has been successfully used to develop the HUST gravity field models [40,41]. The simulation environment is based on numerical orbit integration. The Gauss-Jackson multistep method is used for the numerical integration, which applies start-up values using the Runge-Kutta single step integral method. The adopted gravity field approach is based on the variational equation approach or dynamic method. The orbit is divided into continuous arcs of $6 \mathrm{~h}$ length, and initial state vectors at the begin epoch are set as unknown parameters, which are estimated together with the gravity field coefficients. Kinematic empirical parameters for range-rates are estimated to reduce the 1-cpr effect in the residuals of range-rate observations. The filtered predetermined strategy (FPS) was applied to process these kinematic empirical parameters [40]. No stochastic observation model was used in this simulation work.

Table 3 gives an overview of these methods and the specific settings chosen in the gravity retrieval. 
Table 3. Gravity retrieval methods and specific settings of the 5 processing centers.

\begin{tabular}{|c|c|c|c|c|c|c|}
\hline Proc. Center & Numerical Integration Method & Gravity Retrieval Method & $\begin{array}{l}\text { Max. Degree of } \\
\text { Input Models }\end{array}$ & $\begin{array}{l}\text { Max. Degree of } \\
\text { Retrievals } \\
\text { (Subcycle/Daily) }\end{array}$ & $\begin{array}{c}\text { Co-estimated Parameters (in } \\
\text { addition to Daily } \\
\text { Gravity Fields) }\end{array}$ & Stochastic Model \\
\hline TUM & Adams-Bashforth-Moulton (5 s) & $\begin{array}{l}\text { Integral equations } \\
\text { (short-arc; 6-h arcs) }\end{array}$ & 120 & $70 / 15$ & $\begin{array}{l}\text { Initial position and velocity } \\
\text { (PV) per arc + boundary } \\
\text { condition }\end{array}$ & $\begin{array}{l}\text { Yes, autocovariances } \\
\text { based on residuals }\end{array}$ \\
\hline IGG-CAS & $\begin{array}{c}\text { Gauss Jackson } 12 \\
\text { Start: Runge-Kutta-Nyström 12/10 }\end{array}$ & $\begin{array}{l}\text { Dynamic method } \\
\quad(6-h \text { arcs })\end{array}$ & 70 & $\begin{array}{l}\text { Sc.12: } 70 / 15 \\
\text { Sc.13: } 65 / 15\end{array}$ & Initial PV per arc & No \\
\hline Tongji & $\begin{array}{c}\text { Adams (5 s) and } \\
\text { Kiogh-Shampine-Gordon } 7\end{array}$ & $\begin{array}{l}\text { Integral equations } \\
\text { (short-arc; 2-h arcs) }\end{array}$ & 120 & $70 / 15$ & $\begin{array}{l}\text { Initial positions, daily } \\
\text { polynomials of 5th order, bias } \\
\text { and scale parameter for } \\
\text { acceleration correction per day }\end{array}$ & $\begin{array}{l}\text { Yes, full covariance } \\
\text { based on residuals }\end{array}$ \\
\hline WHU & $\begin{array}{l}\text { Adams-Bashforth-Moulton (5 s) } \\
\text { Start: Runge-Kutta } 8\end{array}$ & $\begin{array}{l}\text { Dynamic method } \\
\text { (1.5-h arcs) }\end{array}$ & 120 & $70 / 15$ & Initial PV per arc & $\begin{array}{l}\text { Yes, ARMA filter } \\
\text { model based on } \\
\text { residuals }\end{array}$ \\
\hline HUST & $\begin{array}{c}\text { Gauss-Jackson } 14 \\
\text { Start: Runge-Kutta } 8\end{array}$ & $\begin{array}{l}\text { Dynamic method } \\
\text { (6-h arcs) }\end{array}$ & 70 & $70 / 15$ & Initial PV per arc & No \\
\hline
\end{tabular}


All processing centers exploit both orbit data for Global Positioning System (GPS) satellite-to-satellite tracking (SST) in high-low mode, as well as the inter-satellite ranging observations (satellite-to-satellite tracking in low-low mode), by adding the corresponding normal equation (NEQ) systems. No additional constraints such as regularization are applied to the NEQ. As shown in Table 3, the methods mainly differ in the co-estimation of additional parameters and the stochastic modelling of instrument errors. WHU, HUST, and IGG-CAS co-parameterize, in addition to daily gravity parameters, only initial state vectors (orbit positions and velocities) per arc. At TUM, additional boundary conditions between arcs are introduced in order to avoid jumps of consecutive arcs. At Tongji, additional daily parameters (polynomials, bias, scale) are co-estimated.

Table 3 shows that the processing centers have used different maximum degree and order for the computation of the observations (70 vs. 120 ), but all of them have resolved gravity fields up to $\mathrm{d} / \mathrm{o} 70$. However, the spectral leakage effect of the signals beyond $\mathrm{d} / \mathrm{o} 70$ is minor compared to the other error sources. If there were a significant spectral leakage effect, it would clearly show up in a severely degraded performance of the very highest resolved degrees [42], which is obviously not the case, as will be shown in Figure 5.

\section{Results and Discussions}

The simulation scenarios described in Chapter 2 have been processed by all contributing groups applying the methods described in Chapter 3 . In this chapter, the results will be compared, assessed, and discussed.

Figure 4 shows the differences of the gravity field solutions of Scenarios A and B to their corresponding references solutions, which are represented by AOHIS mean fields over the 9- and 7-day period, respectively. They are expressed in terms of the degree root mean square (RMS) error of equivalent water heights $(\mathrm{EWH})$, which is computed from fully normalized coefficients of a spherical harmonic series expansion $\left\{\bar{C}_{n m}, \bar{S}_{n m}\right\}$ of degree $n$ and order $m$, by [43]

$$
\sigma_{n}(E W H)=\frac{a \rho_{e}}{3 \rho_{w}} \frac{2 n+1}{1+k_{n}} \sqrt{\sum_{m=0}^{n} \bar{C}_{n m}^{2}+\bar{S}_{n m}^{2}}
$$

where $\rho_{w}$ and $\rho_{e}$ represent the average densities of water and Earth, respectively, $a$ the semi-major axis of the Earth, and $k_{n}$ the load Love number of degree $n .\left\{\bar{C}_{n m}, \bar{S}_{n m}\right\}$ can either represent the full signal, or the differences between estimated and true coefficients. The EWH expresses the height of a mass-equivalent column of water per unit area. It is used here for comparing the results of five processing centres, because it is one of the standard quantities to express mass change processes in the Earth system, and was also used in the reference document [4] for this purpose. Figure 4 shows all 9and 7-daily solutions obtained by the TUM processor for a two-month period as thin lines, as well as the mean of the two-month period as solid lines. Even though Scenario B is based on an inclined satellite with a lower orbit altitude, the performance of these two scenarios is very similar. This can be explained by the fact that Scenario A comprises 9-day solutions, while in Scenario B the solutions are based only on 7 days each. This lower number of observations seems to counteract the lower orbit altitude of the second pair. Since the performance of these two scenarios is very similar, in the following, when we will inter-compare the solutions of the five processing centers, we will concentrate on Scenario A. 


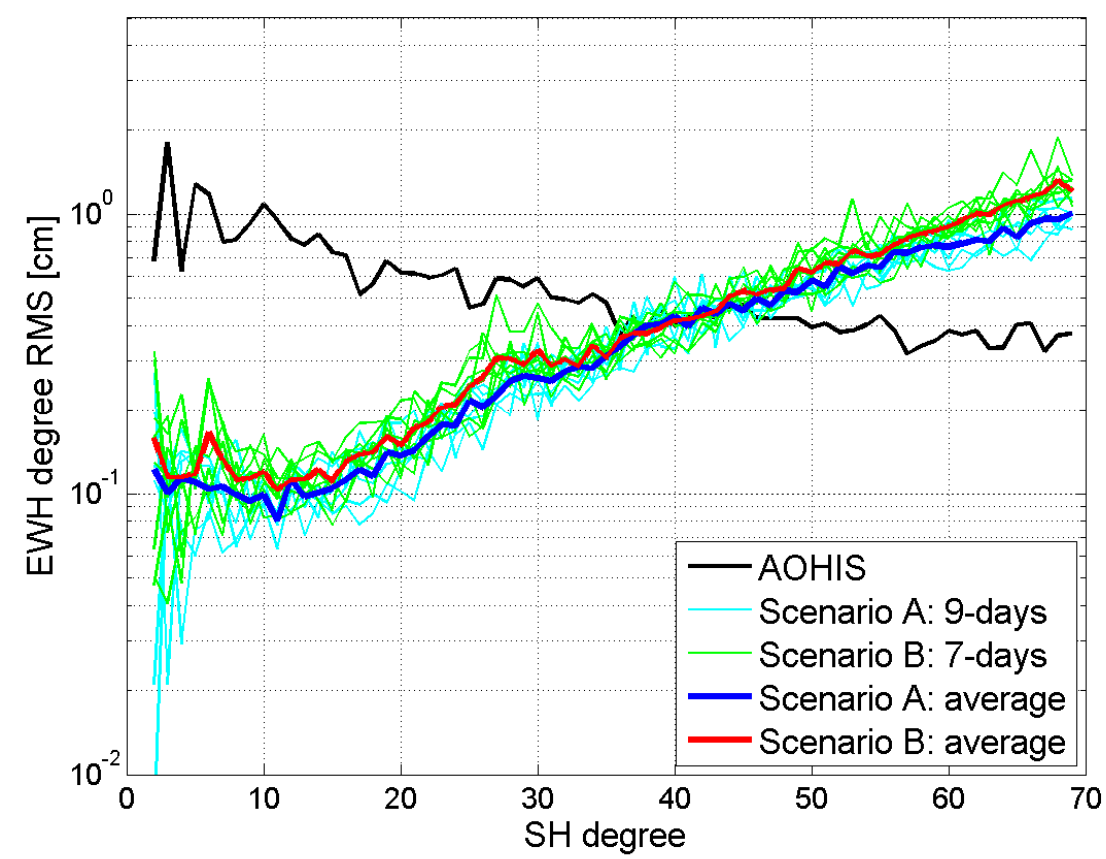

Figure 4. Degree root mean square (RMS) of Scenarios A and B computed with the TUM simulator. The light blue and green lines show the individual 9- and 7-day solutions, respectively, and the blue and red solid lines are the average performance over the two-month period.

Figure 5a shows the degree RMS of the solutions with respect to the AOHIS input signal of the five processing centers for the first 9-day period. Even though there are several differences, they perform reasonably similarly. Apart from a slightly different error level over the whole SH spectrum, evidently the Tongji solutions perform better in the higher degrees, followed by TUM and WHU. A possible reason could be that both processing centers are using full covariance information as a stochastic model (cf. Table 3). In order to analyze the variability of the solutions among themselves, Figure $5 b$ shows again the degree RMS, but now with the TUM solution as the reference. Evidently, the internal variability of the solutions is almost at the same level as their deviation from the input AOHJS signal.

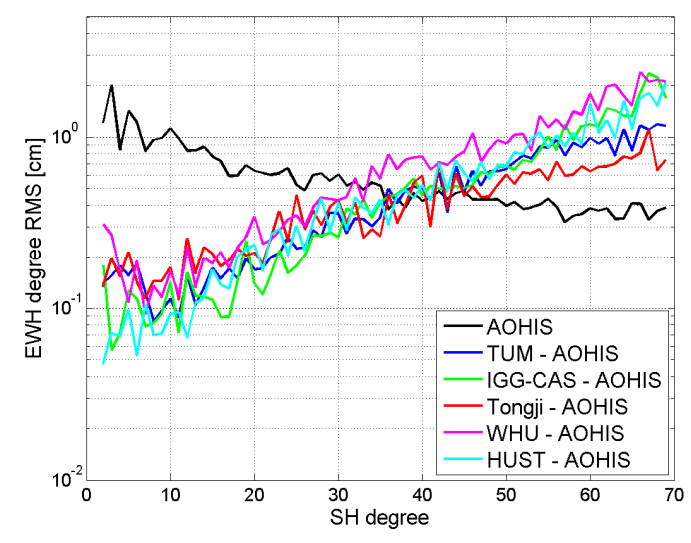

(a)

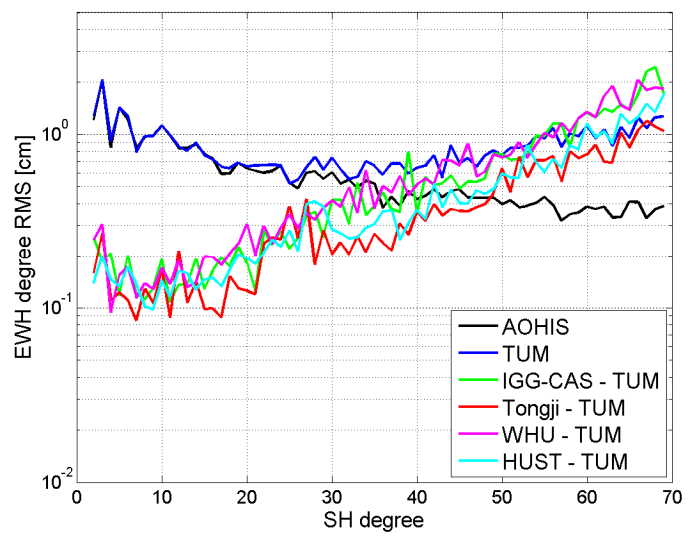

(b)

Figure 5. Degree RMS of first 9-day period of Scenario A of the five numerical simulators: (a) degree RMS with respect to the input AOHIS signal; (b) degree RMS with respect to the TUM solution.

In order to analyze these differences in more detail, Figure 6 shows the SH coefficients of the input AOHIS signal averaged over the first 9 days of the analysis period (Figure 6a), as well as the coefficient differences of the five individual solutions from this AOHIS signal (Figure 6b-f). In general, there is a weakness in the estimation of the sectorial and near-sectorial coefficients, which is intrinsic to 
the in-line inter-satellite ranging concept. It measures mainly in a North-South direction, while the cross-track direction, which is mainly represented by the sectorial coefficients, is determined worse. This general weakness of in-line pairs is also nicely reflected in the estimated formal coefficient standard deviations (Figure 7), representing the main diagonal elements of the variance-covariance matrix.

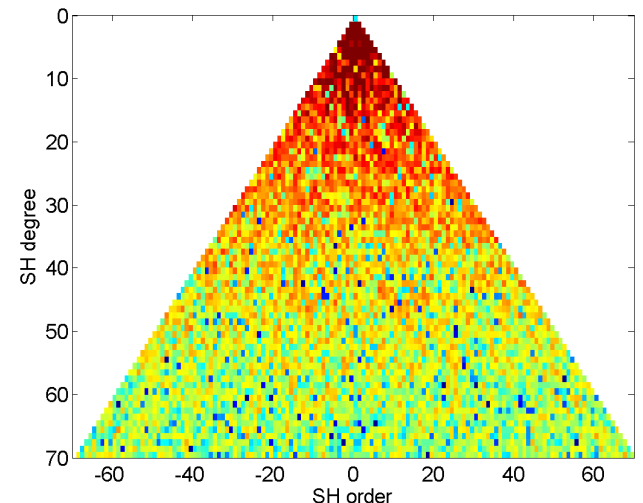

(a)

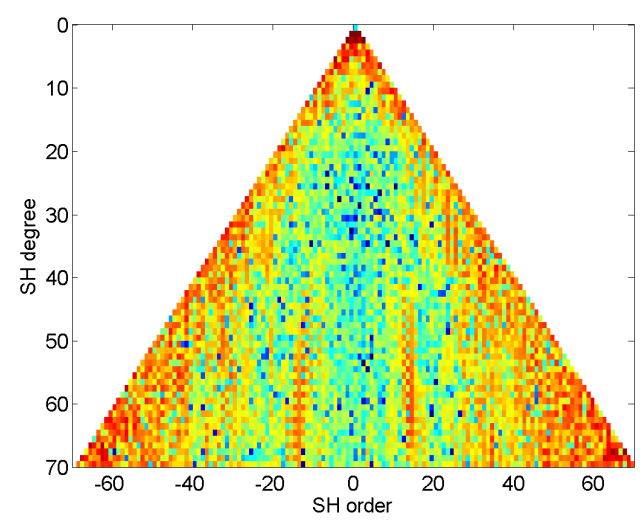

(c)

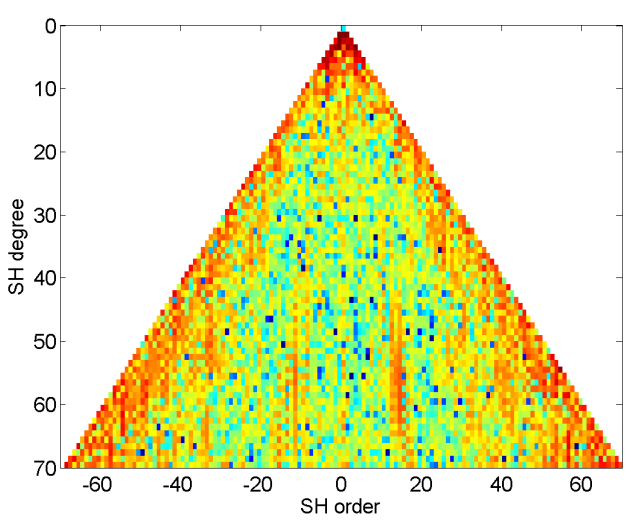

(e)

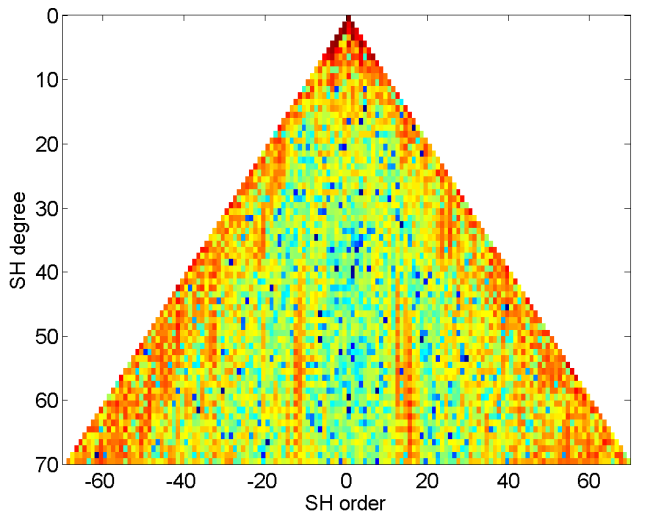

(b)

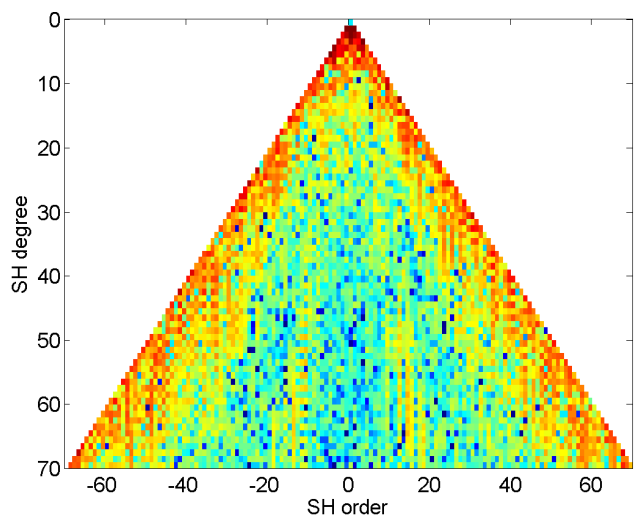

(d)

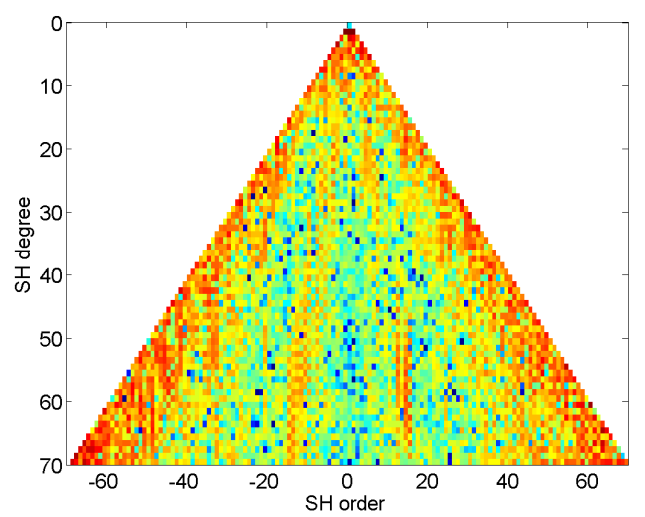

(f)

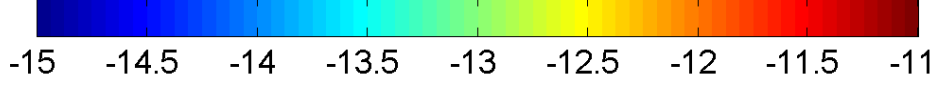

Figure 6. (a) Spherical harmonic (SH) coefficients of the AOHIS model, and SH differences to the AOHIS model of the (b) TUM; (c) IGG-CAS; (d) Tongii; (e) WHU; (f) HUST solutions. Shown is the first 9-day period of Scenario A. Colorbar scale: $\log _{10}(|\ldots|)$. 


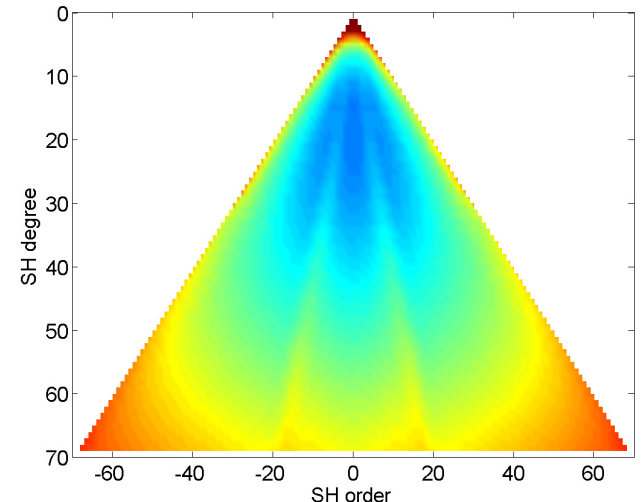

(a)

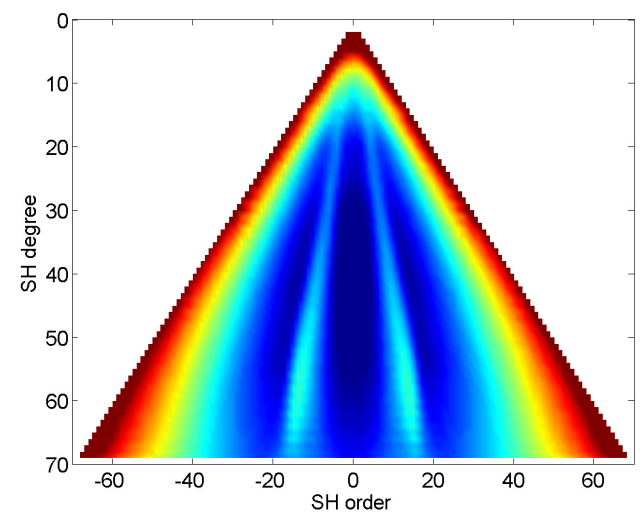

(c)

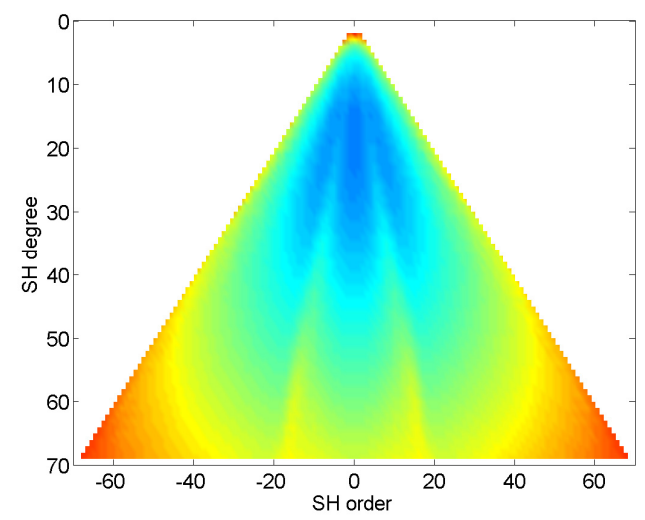

(e)

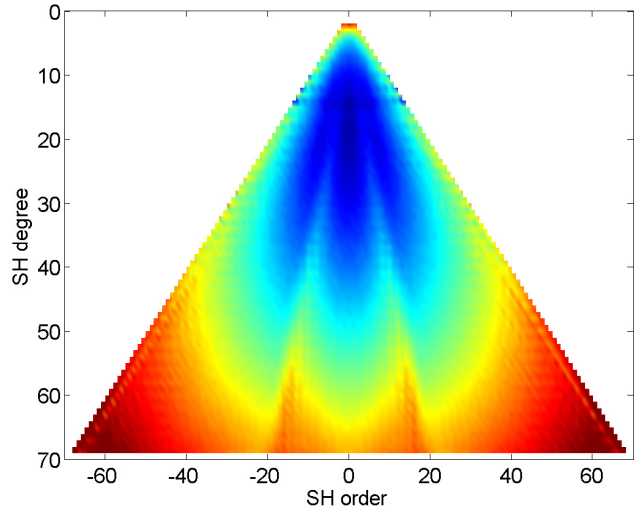

(b)

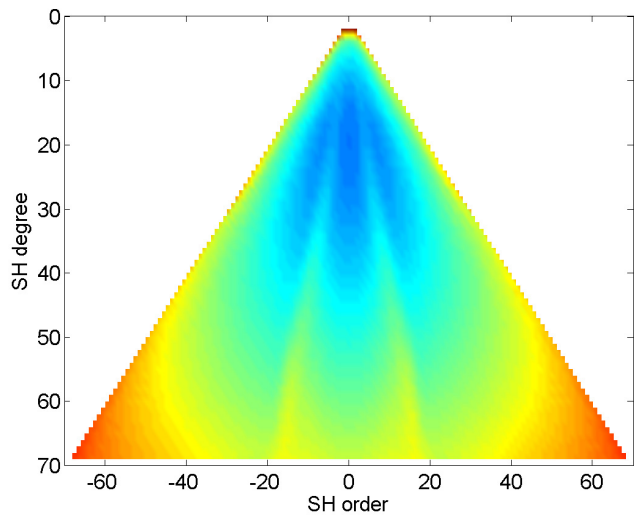

(d)

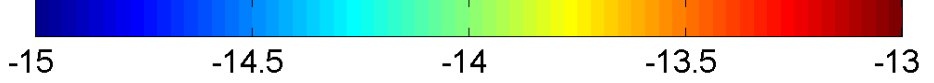

Figure 7. Estimated formal error standard deviations of SH coefficients of the (a) TUM; (b) IGG-CAS; (c) Tongii; (d) WHU; (e) HUST solutions (empirically scaled). Shown is the first 9-day period of Scenario A. Colorbar scale: $\log _{10}(|\ldots|)$.

A typical feature of Bender-type solutions are the error bands forming an inner triangle, which is directly related to the inclination of the second pair, expressing the fact that the near-polar areas are covered only by observations from the polar satellite pair. Since this feature results from the orbit configuration of the double pair, it is also visible in the estimated error standard deviations (Figure 7). In general, these error standard deviations mainly reflect the observation type, the location of the observations, and the stochastic model of observation errors, but they are blind with respect to signal-related temporal aliasing effects, which affect only the right-hand side of the NEQ system. 
An exception would be if these systematic effects are included in the stochastic observation model, as it is done in the Tongji solution. Therefore, the coefficient differences to the true solutions (Figure 6) show a higher error level than the formal error estimates and also additional features, because they are affected by temporal aliasing effects.

In Figure 7, the formal error estimates were scaled by empirically derived factors to make them comparable among each other. This was necessary because the processing centers followed different strategies regarding stochastic modelling (cf. Table 3). Of course, in principle the scaling (calibration) of the formal errors could have been done also by means of a-posteriori variance estimates from the post-fit residuals. However, on the one hand this information was not available for all processing centers, and on the other hand these variance estimates would be hampered by the systematic errors related to temporal aliasing effects anyway.

Analyzing the coefficient differences (Figure 6) and the corresponding scaled statistical error estimates (Figure 7) of the various processing centers in more detail, a striking fact is that the triangular error structure related to the Bender-type constellation of the Tongji solution in Figure $6 \mathrm{~d}$ is much weaker than for the other processing centers, even though it is clearly visible in the error estimate (Figure 7c). This error triangle of the Tongji solution is hardly visible in the coefficient differences to the true AOHIS model of the Tongji solution and looks generally very different from the other solutions (Figure 7c), with much larger degradation of the sectorial and near-sectorial coefficients. As already addressed above, this could be explained by the fact that the covariance information is derived from the post-fit residuals, which contain also temporal aliasing effects. In the IGG-CAS error estimates (Figure 7b), the contrast between the well-estimated near-zonals of lower SH degrees and worse-estimated near-sectorials of high degree is much larger than in the TUM (Figure 7a), WHU (Figure 7d), and HUST (Figure 7e) solutions. Another issue to mention in the error standard deviations is the transition at harmonic degree 15 , which is related to the maximum degree of the daily co-parameterization, which is slightly visible in the IGG-CAS formal errors (Figure 7b), while the other four solutions show a very smooth transition. Analyzing the error estimates of the low-degree $\mathrm{SH}$ coefficients also reveals significant differences. The error estimates of the IGG-CAS (Figure $7 \mathrm{~b}$ ) and HUST (Figure 7e) solutions predict much lower errors of the low-degree components relative to the high-degree coefficients than the other solutions, which might indicate that the GPS-SST high-low component has been given a higher relative weight when combining the NEQs. In this sense, the analysis of the error estimates allows deeper insights into the gravity recovery strategies, leading, however, as discussed above to very comparable results in terms of SH coefficient retrieval.

Figure 8a shows the global EHW field derived from the full AOHIS signal based on the ESA AOHIS model [19] (cf. Table 2) of the first 9-day period of Scenario A, and Figure 8b-f show EWH difference grids associated to the coefficient differences shown in Figure 6. These global grids have been evaluated up to the full $\mathrm{SH}$ resolution of d/o 70. All EWH difference fields show the typical striping pattern which is mainly resulting from residual temporal aliasing. The Tongji and TUM solutions show the lowest stripiness. In order to quantify these results, Table 4 provides an overview of the main statistical parameters of these EWH (difference) fields. Also here, the Tongji solution shows the smallest RMS deviation from the "true" AOHIS model, followed by the TUM result. Evidently, these statistics are dominated by the remaining high-frequency errors expressed by stripes in the EHW fields. Therefore, we re-do the analysis for fields resolved only up to SH d/o 30. According to Figure 5, at this degree the signal-to-noise ratio is safely above one for all five solutions. The results are shown in Figure 9 and Table 5. Please notice that the scale is reduced from $20 \mathrm{~cm}$ to $10 \mathrm{~cm}$ for the EWH (difference) fields. In this d/o 30 analysis, now the IGG-CAS solution performs best, followed by the results of TUM, WHU, and HUST. These three solutions are practically on the same error level. This is also consistent with the degree RMS results shown in Figure 5. 


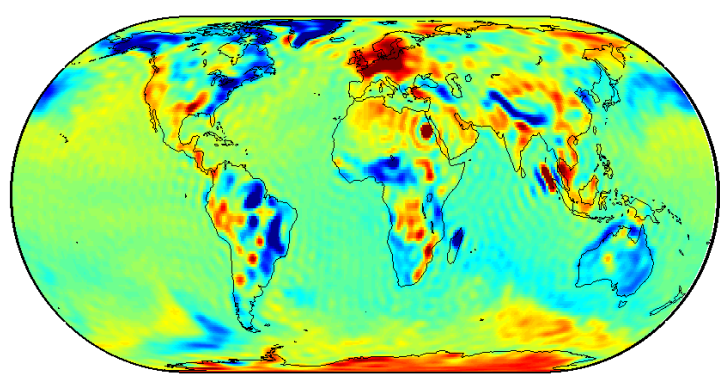

(a)

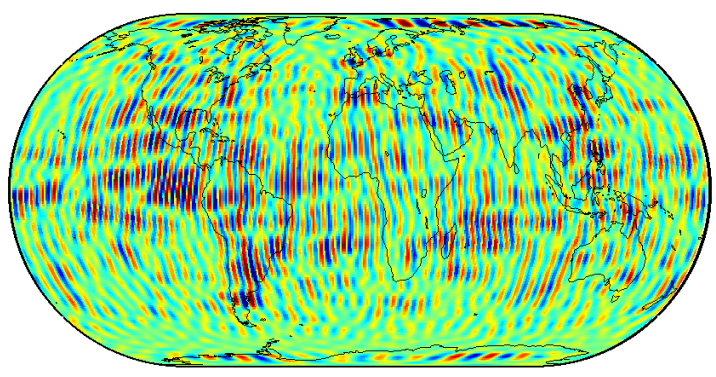

(c)

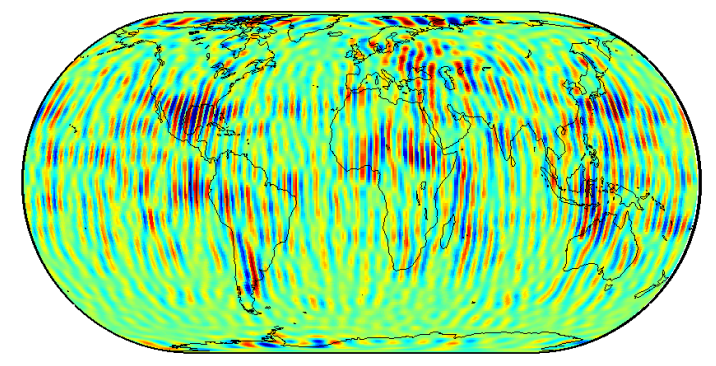

(e)

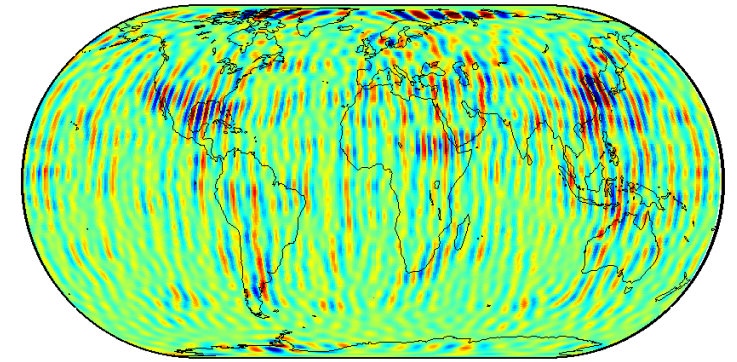

(b)

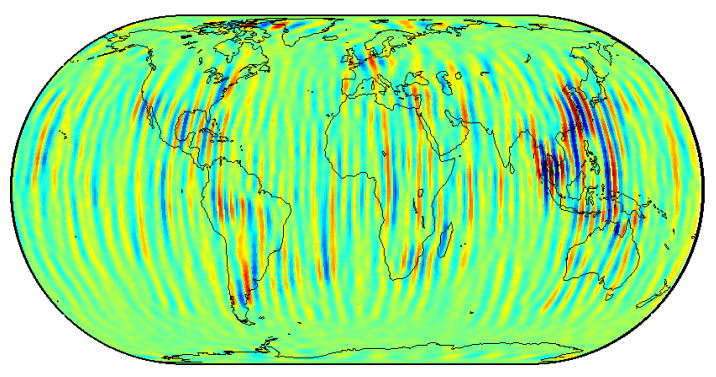

(d)

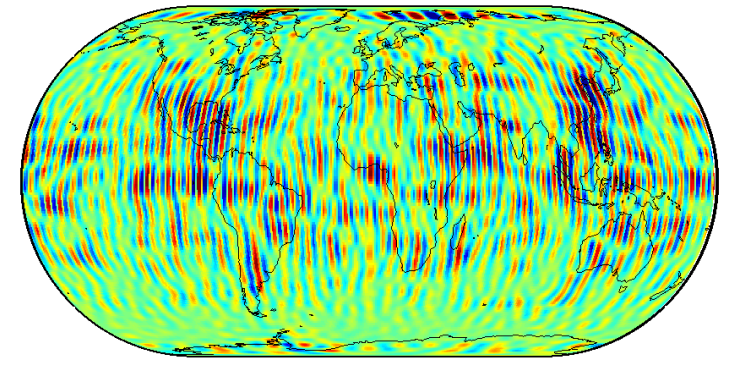

(f)

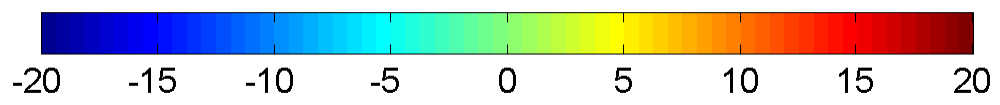

Figure 8. (a) Equivalent water heights $(\mathrm{EWH})(\mathrm{cm})$ grid related to the input AOHIS model up to d/o 70, and EWH (cm) differences to this AOHIS model of the (b) TUM; (c) IGG-CAS; (d) Tongji; (e) WHU; (f) HUST solutions. Shown is the first 9-day period of Scenario A.

Table 4. Main statistical parameters of EWH input and EWH difference fields up to SH d/o 70 of the first 9-day period of Scenario A.

\begin{tabular}{cccc}
\hline Model (d/o 70) & Min $(\mathbf{c m})$ & Max $(\mathbf{c m})$ & RMS (cm) \\
\hline AOHIS & -50.38 & 66.84 & 5.46 \\
TUM - AOHIS & -37.62 & 36.69 & 4.80 \\
IGG-CAS - AOHIS & -29.62 & 30.43 & 6.36 \\
Tongji - AOHIS & -31.46 & 26.71 & 3.91 \\
WHU - AOHIS & -31.97 & 32.58 & 5.41 \\
HUST - AOHIS & -40.68 & 29.56 & 6.11 \\
\hline
\end{tabular}




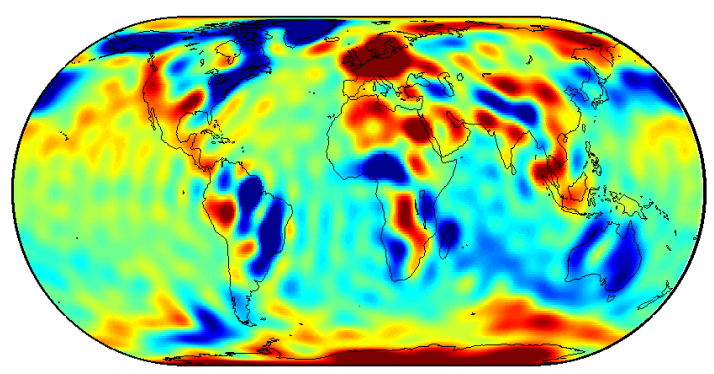

(a)

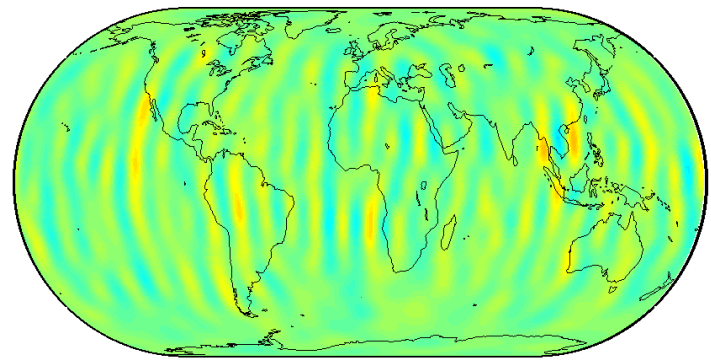

(c)

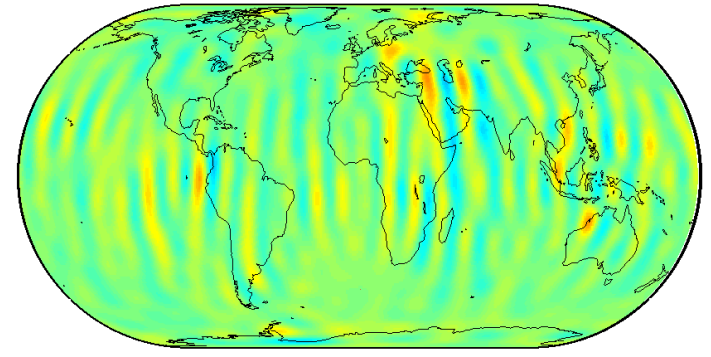

(e)

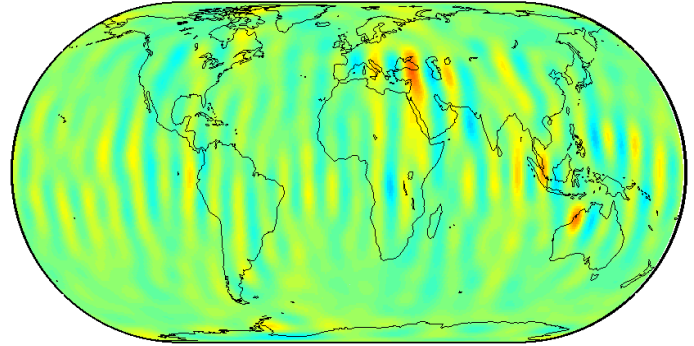

(b)

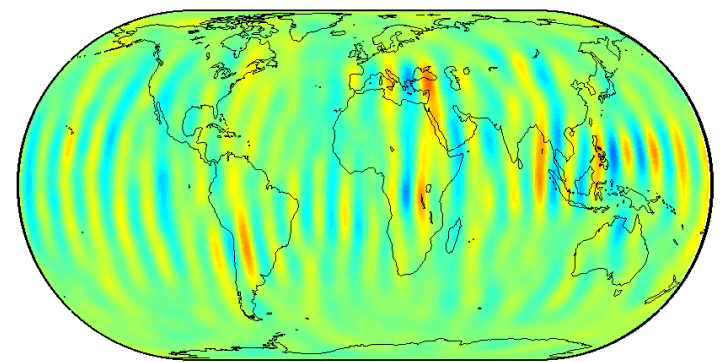

(d)

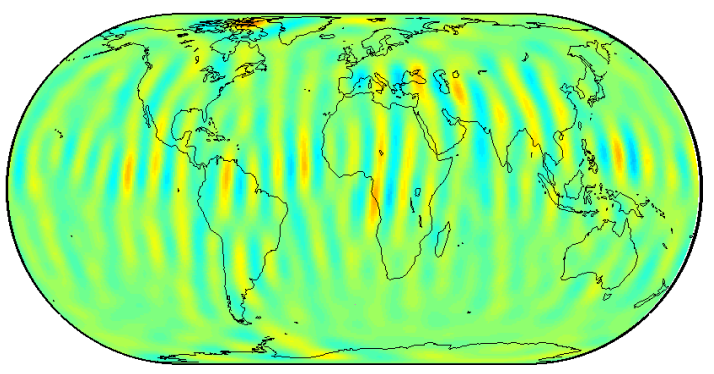

(f)

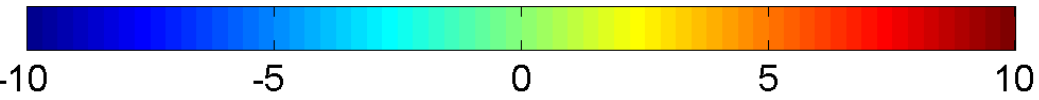

Figure 9. (a) EWH (cm) grid related to the input AOHIS model up to d/o 30, and EWH $(\mathrm{cm})$ differences to this AOHIS model of the (b) TUM; (c) IGG-CAS; (d) Tongii; (e) WHU; (f) HUST solutions. Shown is the first 9-day period of Scenario A.

Table 5. Main statistical parameters of EWH input and EWH difference fields up to SH d/o 30 of the first 9-day period of Scenario A.

\begin{tabular}{cccc}
\hline Model (d/o 30) & Min (cm) & Max (cm) & RMS (cm) \\
\hline AOHIS & -25.83 & 24.90 & 4.74 \\
TUM - AOHIS & -3.63 & 5.42 & 0.98 \\
IGG-CAS - AOHIS & -3.14 & 3.66 & 0.83 \\
Tongji - AOHIS & -5.46 & 5.43 & 1.27 \\
WHU - AOHIS & -3.76 & 4.55 & 1.01 \\
HUST - AOHIS & -3.76 & 4.85 & 1.02 \\
\hline
\end{tabular}

As a general conclusion, at degree 30 the RMS variation among the five solutions is in the order of $10 \%$ of the total signal RMS. Solutions for other time periods show very similar behavior to the ones presented above, and are therefore not explicitly presented and discussed in this paper.

As already mentioned in Chapter 3, all processing centers co-estimated daily gravity field parameters up to $\mathrm{SH}$ d/o 15 in order to reduce temporal aliasing effects. Figure 10 shows the resulting degree error RMS curves, which represent differences of daily estimates to the daily AOHIS "true" signals (black curve), averaged over the whole recovery period. Also here, the performance of these 
daily solutions are very comparable among the processing centers, where IGG-CAS shows a slightly better performance than TUM, HUST, and WHU, and only the Tongji daily estimates are worse.

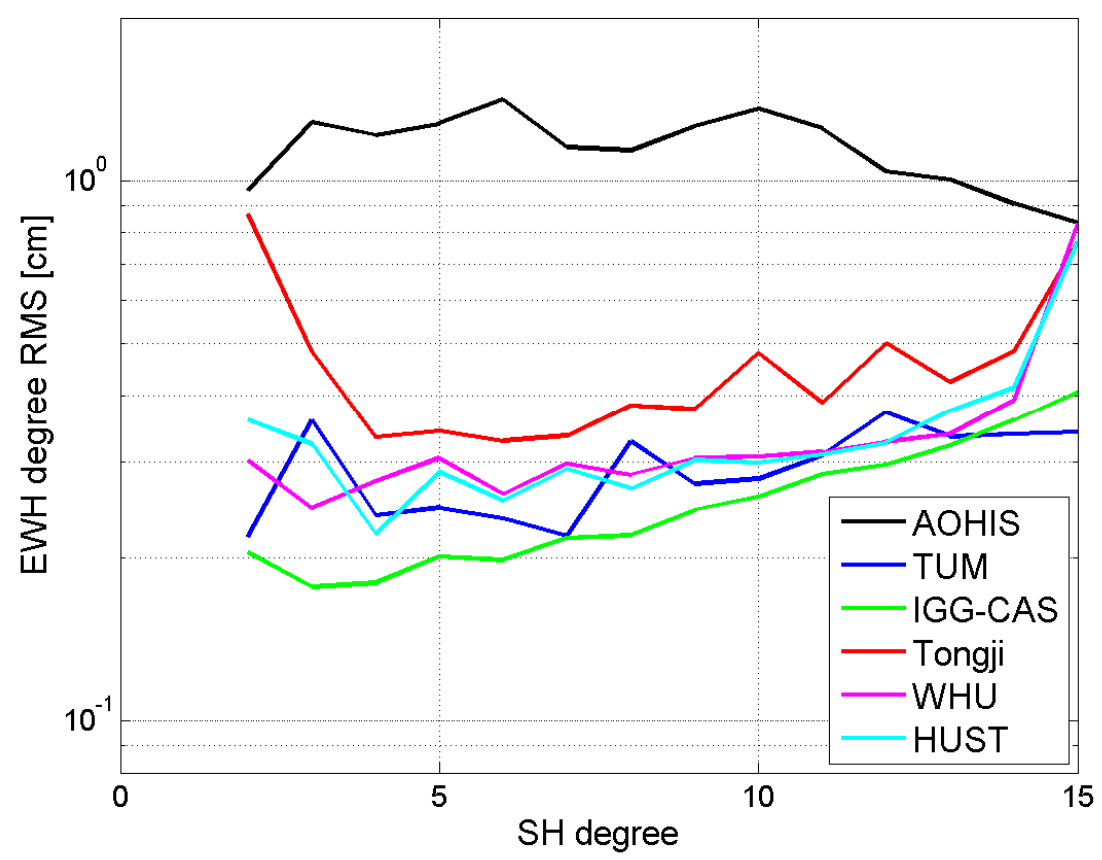

Figure 10. Degree RMS of co-parameterized daily gravity solutions up to d/o 15, averaged over the whole recovery period.

\section{Conclusions and Outlook}

Temporal gravity retrieval results of a future Bender-type double pair mission concept performed by five processing centers of a Sino-European NGGM study team based on jointly defined mission scenarios have been inter-compared and assessed. In spite of some remaining differences, the solutions of the contributing teams TU Munich, Institute of Geodesy and Geophysics, Tongji University, Wuhan University, and Huazhong University of Science and Technology show quite similar performances. In general, these results demonstrate that one of the main goals of this inter-comparison exercise, the consistency of simulation results among the contributing groups, could be achieved to a large extent. Remaining differences might be explained by different processing strategies and partially different parameterizations, such as the co-parameterization of accelerometer biases and drifts, or empirical parameters to compensate for long-wavelength errors in the observation time series. Some remaining differences also exist in the error level of the co-estimated daily solutions.

As already mentioned above, the main study goal was not to identify a "winner" among the contributing groups, but rather to achieve consistency of numerical simulation results of NGGM concepts to the best possible extent, even though five independent software packages, which are based partly on different gravity retrieval methods, have been applied. This positive result is an important pre-requisite and basis for future joint activities towards the realization of NGGMs.

Based on this successful inter-comparison exercise of numerical simulators and in view of the fact that in the future several satellite pairs might be in orbit in parallel, further joint studies on optimized constellations of triple- and multi-pairs will be performed, and their impact on the main fields of applications, such as hydrology, glaciology, and solid Earth physics, will be quantified. The results of these studies will be presented in a follow-up paper.

Author Contributions: Conceptualization, R.P., H.-C.Y., W.F., Z.L., Y.S., M.Z., H.X.; Methodology, R.P., Q.C., M.H., A.P., X.X., B.Z., H.Z., X.Z.; Software, Q.C., M.H., A.P., X.X., B.Z., H.Z., B.L., Y.Z., X.Z.; Validation of results, A.P., R.P.; Writing—original draft preparation, R.P.; Writing—review and editing, R.P., Q.C., W.F., R.H., M.H., Z.L., A.P., Y.S., C.W., X.X., B.Z., H.Z., B.L., Y.Z., X.Z., H.Y., M.Z.; visualization, R.P. 
Funding: This research was performed in the framework of the study "Assessment of satellite constellations for monitoring the variations in Earth gravity field (ADDCON)", ESA-ESTEC, Contract AO/1-7317/12/NL/AF funded by the European Space Agency. This study was also funded by the National Natural Science Foundation of China (projects no. 41704012, 41931074 and 41731069), and the Strategic Priority Research Program of the Chinese Academy of Sciences (grant nos. XDB23030100 and XDA15017700).

Acknowledgments: We further acknowledge the contribution of the ADDCON project partners Pieter Visser, Nicolas Sneeuw, Wei Liu, Johannes Engels, Qiang Chen, Tonie van Dam and Thomas Gruber for a successful completion of the ESA project, as well as Christian Siemes and Luca Massotti for their valuable support during this project.

Conflicts of Interest: The authors declare no conflict of interest.

\section{References}

1. Tapley, B.D.; Bettadpur, S.; Watkins, M.; Reigber, C. The gravity recovery and climate experiment experiment, mission overview and early results. Geophys. Res. Lett. 2004, 31. [CrossRef]

2. Flechtner, F.; Neumayer, K.H.; Dahle, C.; Dobslaw, H.; Fagiolini, E.; Raimondo, J.C.; Günter, A. What can be expected from the GRACE-FO Laser Ranging Interferometer for Earth Science applications? Surv. Geophys. 2016, 37, 453-470. [CrossRef]

3. Drinkwater, M.R.; Floberghagen, R.; Haagmans, R.; Muzi, D.; Popescu, A. GOCE: ESA's first Earth Explorer Core mission. In Earth Gravity Field from Space - from Sensors to Earth Science; Space Sciences Series of ISSI; Beutler, G., Drinkwater, M.R., Rummel, R., Steiger, R., Eds.; Kluwer Academic: Dordrecht, The Netherlands, 2003; Volume 18, pp. 419-432. ISBN 1-4020-1408-2.

4. Pail, R.; Bingham, R.; Braitenberg, C.; Dobslaw, H.; Eicker, A.; Güntner, A.; IUGG Expert Panel. Science and user needs for observing global mass transport to understand global change and benefit society. Surv. Geophys. 2014, 36, 743-772. [CrossRef]

5. Murböck, M.; Pail, R.; Daras, I.; Gruber, T. Optimal orbits for temporal gravity recovery regarding temporal aliasing. J. Geod. 2015, 88, 113-126. [CrossRef]

6. Panet, I.; Flury, J.; Biancale, R.; Gruber, T.; Johannessen, J.; van den Broeke, M.R.; van Dam, T.; Gegout, P.; Hughes, C.W.; Ramillien, G.; et al. Earth System Mass Transport Mission (e.motion): A Concept for future Earth Gravity Field Measurements from Space. Surv. Geophys. 2012, 34, 141-163. [CrossRef]

7. Bender, P.L.; Wiese, D.N.; Nerem, R.S. A possible Dual-GRACE mission with 90 degree and 63 degree inclination orbits. In Proceedings of the 3rd International Symposium on Formation Flying, Missions and Technologies, Noordwijk, The Netherlands, 23-25 April 2008.

8. Wiese, D.N.; Nerem, R.S.; Han, S.C. Expected improvements in determining continental hydrology, ice mass variations, ocean bottom pressure signals, and earthquakes using two pairs of dedicated satellites for temporal gravity recovery. J. Geophys. Res. Solid Earth 2011, 116. [CrossRef]

9. Daras, I.; Pail, R. Treatment of temporal aliasing effects in the context of next generation satellite gravimetry missions. J. Geophys. Res. 2017, 122, 7343-7362. [CrossRef]

10. Gruber, T.; Panet, I.; E.motion2 Team. Proposal to ESA's Earth Explorer Call 9: Earth System Mass Transport Mission-E.motion2. Deutsche Geodätische Kommission der Bayerischen Akademie der Wissenschaften, Reihe B, Angewandte Geodäsie, Series B. 2015. Available online: https://dgk.badw.de/fileadmin/user_upload/ Files/DGK/docs/b-318.pdf (accessed on 12 November 2019).

11. Pail, R.; Bamber, J.; Biancale, R.; Bingham, R.; Braitenberg, C.; Eicker, A.; Flechtner, F.; Gruber, T.; Güntner, A.; Heinzel, G.; et al. Mass variation observing system by high low inter-satellite links (MOBILE)—a new concept for sustained observation of mass transport from space. J. Geod. Sci. 2019. [CrossRef]

12. Hauk, M.; Pail, R. Gravity field recovery by high-precision high-low inter-satellite links. Remote Sens. 2019, 11, 537. [CrossRef]

13. NAS-National Academies of Sciences, Engineering, and Medicine. Thriving on Our Changing Planet: A Decadal Strategy for Earth Observation from Space; The National Academies Press: Washington, DC, USA, 2018. Available online: https://essp.nasa.gov/essp/files/2018/02/2017-Earth-Science-Decadal-Survey.pdf (accessed on 12 November 2019).

14. Lu, Y.; Li, H.; Yeh, H.C.; Luo, J. A self-analyzing double-loop digital controller in laser frequency stabilization for inter-satellite laser ranging. Rev. Sci. Instrum. 2015, 86, 044501. [CrossRef] 
15. Liang, Y.R.; Duan, H.Z.; Xiao, X.L.; Wei, B.B.; Yeh, H.C. Inter-satellite laser range-rate measurement by using digital phase locked loop. Rev. Sci. Instrum. 2015, 86, 016106. [CrossRef] [PubMed]

16. Zhang, J.Y.; Ming, M.; Jiang, Y.Z.; Duan, H.Z.; Yeh, H.C. Inter-satellite laser link acquisition with dual-way scanning for Space Advanced Gravity Measurements mission. Rev. Sci. Instrum. 2018, 89, 064501. [CrossRef] [PubMed]

17. Luo, J.; Chen, L.S.; Duan, H.Z.; Gong, Y.G.; Hu, S.; Ji, J.; Liu, Q.; Mei, J.; Milyukov, V.; Sazhin, M.; et al. TianQin: A space-borne gravitational wave detector. Class. Quantum Gravity 2016, 33, 035010. [CrossRef]

18. Mayer-Gürr, T.; Pail, R.; Gruber, T.; Fecher, T.; Rexer, M.; Schuh, W.D.; Kusche, J.; Brockmann, J.M.; Rieser, D.; Zehentner, N.; et al. The combined satellite gravity field model GOCO05s. Geophysical Research Abstracts, EGU2015-12364. In Proceedings of the European Geosciences Union General Assembly 2015, Vienna, Austria, 12-17 April 2015; Volume 17.

19. Dobslaw, H.; Flechtner, F.; Bergmann-Wolf, I.; Dahle, C.; Dill, R.; Esselborn, S.; Sasgen, I.; Thomas, M. Simulating high-frequency atmosphere-ocean variability for dealiasing of satellite gravity observations: AOD1b RL05: ATMOSPHERE-OCEAN MASS VARIABILITY: AOD1b. J. Geophys. Res. Oceans 2013, 118, 3704-3711. [CrossRef]

20. Savcenko, R.; Bosch, W. EOT11A_Empirical Ocean Tide Model from Multi-Mission Satellite Altimetry; Deutsches Geodätisches Forschungsinstitut (DGFI): München, Germany, 2012.

21. Ray, R.D. A Global Ocean Tide Model from Topex/Poseidon Altimetry: Got99.2; Technical Report; NASA Technical Memorandum 209478; NASA: Washington, DC, USA, 1999.

22. Iran Pour, S.; Reubelt, T.; Sneeuw, N.; Daras, I.; Murböck, M.; Gruber, T.; Pail, R.; Weigelt, M.; van Dam, T.; Visser, P.; et al. Assessment of Satellite Constellations for Monitoring the Variations in Earth Gravity Field—SC4MGV; ESA-ESTEC Contract No. AO/1-7317/12/NL/AF, Final Report; University of Stuttgart: Stuttgart, Germany, 2015.

23. Wiese, D.N.; Visser, P.; Nerem, R.S. Estimating low resolution gravity fields at short time intervals to reduce temporal aliasing errors. Adv. Space Res. 2011, 48, 1094-1107. [CrossRef]

24. Purkhauser, A.; Pail, R. Next generation gravity missions: Near-real time gravity field retrieval strategy. Geophys. J. Int. 2019, 217. [CrossRef]

25. Daras, I.; Pail, R.; Murböck, M.; Yi, W. Gravity field processing with enhanced numerical precision for LL-SST missions. J. Geod. 2015, 89, 99-110. [CrossRef]

26. Daras, I. Gravity Field Processing Towards Future LL-SST Satellite Missions; DGK Series C, Dissertation No. 770; Verlag der Bayerischen Akademie der Wissenschaften: Munich, Germany, 2016; pp. 23-39. ISSN 0065-5325.

27. Shampine, L.F.; Gordon, M.K. Computer Solution of Ordinary Differential Equations: The Initial Value Problem; W.H. Freeman: San Francisco, CA, USA, 1975.

28. Schneider, M. Outline of a general orbit determination method. In Space Research IX, Proceedings of the Open Meetings of Working Groups (OMWG) on Physical Sciences of the 11th Plenary Meeting of the Committee on Space Research (COSPAR), Tokyo, Japan, 9-21 May 1968; Mitteilungen aus dem Institut für Astronomische und Physikalische Geodäsie, Nr. 51; Champion, K.S.W., Smith, P.A., Smith-Rose, R.L., Eds.; North Holland Publ. Company: Amsterdam, The Netherlands, 1969; pp. 37-40.

29. Wang, C.; Xu, H.; Zhong, M.; Feng, W. Monthly gravity field recovery from GRACE orbits and K-band measurements using variational equations approach. Geod. Geodyn. 2015, 6, 253-260. [CrossRef]

30. Berry, M.M.; Healy, L.M. Implementation of Gauss-Jackson Integration for Orbit Propagation. J. Astronaut. 2004, 52, 331-357.

31. Kim, J. Simulation Study of a Low-Low Satellite-to-Satellite Tracking Mission. Ph.D. Thesis, The University of Texas at Austin, Austin, TX, USA, 2000.

32. Chen, Q.; Shen, Y.; Zhang, X.; Hsu, H.; Chen, W.; Ju, X.; Lou, L. Monthly gravity field models derived from GRACE level 1b data using a modified short arc approach. J. Geophys. Res. Solid Earth 2015, 120, 1804-1819. [CrossRef]

33. Chen, Q.; Shen, Y.; Chen, W.; Zhang, X.; Hsu, H. An improved GRACE monthly gravity field solution by modeling the non-conservative acceleration and attitude observation errors. J. Geod. 2016, 90, 503-523. [CrossRef]

34. Chen, Q.; Shen, Y.; Francis, O.; Chen, W.; Zhang, X.; Hsu, H. Tongji-Grace02s and Tongji-Grace02k: High-precision static GRACE-only global Earth's gravity field models derived by refined data processing strategies. J. Geophys. Res. Solid Earth 2018, 123, 6111-6137. [CrossRef] 
35. Chen, Q.; Shen, Y.; Chen, W.; Francis, O.; Zhang, X.; Chen, Q.; Li, W.; Chen, T. An optimized short-arc approach: Methodology and application to develop refined time series of Tongji-Grace2018 GRACE monthly solutions. J. Geophys. Res. 2019, 124, 6010-6038. [CrossRef]

36. Zou, X.C.; Zhong, L.P.; Li, J.C. Simultaneous solution for precise satellite orbit and earth gravity model. Chin. J. Geophys. 2016, 59, 2413-2423.

37. Xu, X.; Zhao, Y.; Reubelt, T.; Tenzer, R. A GOCE only gravity model GOSG01S and the validation of GOCE related satellite gravity models. Geod. Geodyn. 2017, 8, 260-272. [CrossRef]

38. Reigber, C. Gravity field recovery from satellite tracking data. In Theory of Satellite Geodesy and Gravity Field Determination; Lecture Notes in Earth Sciences; Sansò, F., Rummel, R., Eds.; Springer: Berlin/Heidelberg, Germany, 2005; Volume 25, pp. 197-234.

39. Schuh, W.D. The processing of band-limited measurements; filtering techniques in the least squares context and in the presence of data gaps. Space Sci. Rev. 2003, 108, 67-78. [CrossRef]

40. Zhou, H.; Luo, Z.; Zhou, Z.; Li, Q.; Zhong, B.; Lu, B.; Hsu, H. Impact of different kinematic empirical parameters processing strategies on temporal gravity field model determination. J. Geophys. Res. Solid Earth 2018, 123, 252-276. [CrossRef]

41. Zhou, H.; Zhou, Z.; Luo, Z. A new hybrid processing strategy to improve temporal gravity field solution. J. Geophys. Res. Solid Earth 2019, 124. [CrossRef]

42. Goossens, S. Applying spectral leakage corrections to gravity field determination from satellite tracking data. Geophys. J. Int. 2010, 181, 1459-1472. [CrossRef]

43. Wahr, J.; Moleneer, M.; Bryan, F. Time variability of the Earth's gravity field: Hydrological and oceanic effects and their possible detection using GRACE. J. Geophys. Res. Solid Earth 1998, 103, 30205-30229. [CrossRef]

(C) 2019 by the authors. Licensee MDPI, Basel, Switzerland. This article is an open access article distributed under the terms and conditions of the Creative Commons Attribution (CC BY) license (http://creativecommons.org/licenses/by/4.0/). 